\title{
SOME REMARKS ON THE KRONHEIMER-MROWKA CLASSES OF ALGEBRAIC SURFACES
}

\author{
ROGIER BRUSSEE
}

\section{Introduction}

Recently Kronheimer and Mrowka have announced a very interesting result, which sheds new light on the Donaldson polynomials [8]. They find recurrence relations between the Donaldson polynomials, and a relation between the polynomials and the minimal genus of a smooth real surface representing a homology class. To be more precise we need a definition.

For a simply connected 4-manifold $X$ with odd $b_{+} \geq 3$, we denote the $\mathrm{SU}(2)$ polynomials on $H_{0}(X) \oplus H_{2}(X)$ by $q_{k}(X) . X$ is said to be of simple type if

$$
q_{k}(X)(\mathrm{pt}, \mathrm{pt},-)=4 q_{k-1}(X), \quad d=4 k-\frac{3}{2}\left(1+b_{+}\right) .
$$

For 4-manifolds of simple type it is convenient to label the polynomials by their degree on $\mathrm{H}_{2}(X)$, i.e., we define

$$
q_{d}(X)= \begin{cases}\left.q_{k}(X)\right|_{H_{2}(X)} & \text { if } d=4 k-\frac{3}{2}\left(1+b_{+}\right), \\ \left.\frac{1}{2} q_{k}(X)(p t,-)\right|_{H_{2}(X)} & \text { if } d=4 k-2-\frac{3}{2}\left(1+b_{+}\right), \\ 0 & \text { otherwise }\end{cases}
$$

The Donaldson series is then the formal power series $q(X)=\sum_{d} q_{d}(X) / d$ !.

Theorem 1 (Kronheimer-Mrowka). For every simply connected 4-manifold Xof simple type there exist Kronheimer-Mrowka classes $K_{1}, \ldots, K_{p} \in$ $H^{2}(X)$ and nonzero rational numbers $a_{1}, \ldots, a_{p}$ such that

(i) $q(X)=e^{Q / 2} \sum_{i=1}^{p} a_{i} e^{K_{i}}$,

(ii) $K_{i} \equiv w_{2}(X)(\bmod 2)$, for all $i=1, \ldots, p$,

(iii) if $K_{i} \in\left\{K_{1}, \ldots, K_{p}\right\}$, then $-K_{i} \in\left\{K_{1}, \ldots, K_{p}\right\}$,

Received September 14, 1993. The author is grateful for support by the Graduirtenkolleg Komplexe Mannigfaltigkeiten Bayreuth, where this article was written. 
(iv) for every homologically nontrivial connected real surface $\Sigma$ with $\Sigma^{2} \geq 0$ and every Kronheimer-Mrowka class $K_{i}$ we have

$$
2 g(\Sigma)-2 \geq \Sigma^{2}+K_{i} \cdot \Sigma .
$$

Here $Q$ is the intersection form. The Kronheimer-Mrowka classes are called the basic classes in [8] and will be abbreviated to KM classes. Condition (ii) is reminiscent of the $\mathrm{Wu}$ formula, whereas condition (iv) is similar to the adjunction formula for the genus of a smooth algebraic curve. This suggests that for complex surfaces, the KM classes should be closely related to the canonical divisor $K_{X}$. Indeed in the examples and the expression for the Donaldson series of elliptic surfaces conjectured in the announcement, and proved by Fintushel and Stern [3], the KM classes are of type $K_{i}=\alpha_{i} K_{\min }+\sum \beta_{i j} E_{j}$ where $K_{\min }$ is the canonical divisor of the minimal model, $E_{1}, \ldots, E_{l}$ are the $(-1)$-curves, $\alpha_{i}$ is a rational number with $\left|\alpha_{i}\right| \leq 1$ and $\beta_{i j}= \pm 1$. A formulation, which is a little less obvious but, as we will see, generalizes better, is $K_{i}=C-D$, where $C$, $D$ are divisors such that $K_{X}=C+D$ and such that a multiple is effective. Hence in these cases the canonical class is a KM class which is extremal from an algebraic geometric point of view.

To formulate the general relationship and our main theorem, we need a definition. The effective cone $\mathrm{NE}(X)$ of a complex surface is the positive rational cone in $\mathrm{NS}(X)_{\mathbb{Q}} \subset H^{2}(X, \mathbb{Q})$ generated by the effective divisors. The Mori cone $\overline{\mathrm{NE}}(X)$ is the closure $\mathrm{NE}(X)$ in the norm topology.

Theorem 2. For every $K M$ class $K_{i}$ on a simply connected algebraic surface $X$ of simple type with $p_{g}>0$, there is a unique decomposition $K_{X}=C_{i}+D_{i}$ in $\mathbb{Z}$-divisors $C_{i}, D_{i} \in \overline{N E}(X)$ such that $K_{i}=C_{i}-D_{i}$. In particular, $K_{i}=K_{X}$ if and only if there is a smooth hyperplane section $H$ such that $2 g(H)-2=H^{2}+K_{i} \cdot H$.

For minimal surfaces of general type, the canonical class is numerically connected [1, Proposition VII.6.1]. Since in the above theorem $C_{i}, D_{i} \in$ $\overline{\mathrm{NE}}(X)$, we find that the intersection $C_{i} \cdot D_{i} \geq 0$ with equality if and only if $C_{i}$ or $D_{i}=0$ (strictly speaking, in the definition of numerical connectedness we demand that $C_{i}$ and $D_{i}$ are effective, but the proof goes through without change). Thus writing $K_{i}^{2}=K_{X}^{2}-4 C_{i} \cdot D_{i}$ we find

Corollary 3. Assume in addition that $X$ is minimal and of general type, then $K_{i}^{2} \leq K_{X}^{2}$ with equality if and only if $K_{i}= \pm K_{X}$.

Kronheimer's result on the generalised Thom conjecture [7] implies that a smooth hyperplane section $H$ has minimal genus in its homology class, at least when there is an $\omega \in H^{0}(K)$ such that for sufficiently large $k$, 
$q_{k}(\omega+\bar{\omega}) \neq 0$. Since this result is based on the same techniques as the proof of the existence of the KM classes, Theorem 2 strongly suggests that the canonical class is among the $\mathrm{KM}$ classes, at least under the technical conditions mentioned and the simple type condition. ${ }^{1}$ Then by Corollary 3 , for minimal surfaces of general type, the canonical class is a $C^{\infty}$ invariant up to sign (alternatively invariance would follow if it is true that the $\mathrm{KM}$ classes define an almost complex structure, i.e., if $\left.K_{i}^{2}=3 \operatorname{sign}+2 \chi_{\text {top }}\right)$. For minimal elliptic surfaces the invariance up to sign is equivalent to the invariance of the multiplicities of the multiple fibers, and the invariance of the subspace of $\mathrm{H}_{2}$ spanned by the fiber. This is a by-now well-known fact, which follows, for example, from the formula for their Donaldson series. Thus, barring the question how general the simple type condition is, in the case $p_{g}>0$ and assuming simply connectedness, the Kronheimer-Mrowka formalism seems to explain the observed invariance of the canonical class of a minimal surface. Finally the suggested blow-up formula [8, formula (4) f.f.] and a differentiable characterization of $(-1)$-curves [4], [2] would extend this discussion to the nonminimal case.

By the Lefschetz $(1,1)$ theorem [6, p. 163], the algebraicity of the KM classes is equivalent to the $K_{i}$ being of type $(1,1)$. As in [2] this turns out to be a formal consequence of the fact that the Donaldson polynomials are of pure Hodge type. Since we assume that $p_{g}>0$, this shows that the lattice spanned by the $K_{i}$ is a proper sublattice of $H^{2}(X)$. Moreover since the $K_{i}$ are defined by the differentiable structure, they are contained in the fixed lattice of the variation of Hodge structures defined by a family of complex structures on $X$. In favorable circumstances this should force the $\mathrm{KM}$ classes to be in $H^{2}(X, \mathbb{Z}) \cap\left[K_{\min }, E_{1}, \ldots, E_{l}\right]$.

Another implication of the theorem is that the KM classes are trivial on $H^{0,2} \subset H^{2}(X, \mathbb{C})$. Thus we get the following corollary.

Corollary 4. If $X$ is a simple and simply connected surface, then for all $\omega \in H^{0}\left(K_{X}\right)$ we have

$$
q(\omega+\bar{\omega})=q_{0} e^{\int \omega \wedge \bar{\omega}},
$$

where $q_{0}$ is the Donaldson polynomial of degree 0 .

It would be rather interesting to understand this formula from an algebraic geometric point of view, possibly clarifying the role of the simpleness condition. Combining Corollary 4 with O'Grady's nonvanishing result, we

\footnotetext{
${ }^{1}$ Added in proof. Kronheimer has informed the author that the canonical class is indeed a KM class if the surface is of simple type and $q_{k}(\omega+\bar{\omega}) \neq 0$ for $k \gg 0$.
} 
see that $q_{0} \neq 0$ if $X$ is of general type, $p_{g}$ is odd and $\left|K_{\min }\right|$, the linear system of the canonical class of the minimal model, contains a reduced curve [13, Theorem 2.4], [10, Appendix], [11, Theorem 1].

In a similar vein, since the Neron-Severi group has a nondegenerate intersection form, the $K_{i}$ are determined by their intersection products with divisors. Thus we get

Corollary 5. For a simply connected surface of simple type, the Donaldson series $q(X)$ is determined by $\left.q(X)\right|_{\mathrm{NS}(X)}$.

Corollary 5 states that by knowing the algebraic part of all Donaldson polynomials we can reconstruct the transcendental part as well, i.e., in the simple type case, the polynomials defined by J. Li [9] contain as much information as the full polynomials. Moreover since $\overline{\mathrm{NE}} \cap K_{X}-\overline{\mathrm{NE}}$ is a bounded subset of $\mathrm{NS}(X, \mathbb{Q})$, and the $K_{i}$ are integral, at least in principle, we get an effective bound on the number of Kronheimer-Mrowka classes, hence on the number of polynomials we have to compute in order to reconstruct the Donaldson series.

\section{Proof of Theorem 2}

We have to prove that the $\mathrm{KM}$ classes are of type $(1,1)$. Accepting this claim for a moment, the rest of the statement of Theorem 2 is a consequence of properties (ii), (iii), and (iv) of the KM classes.

By property (ii) we can write $K_{i}=K_{X}-2 D$ for some $\mathbb{Z}$-divisor $D$. For every very ample line bundle $\mathscr{O}(H)$, we choose a smooth connected hyperplane section $H$. Then we get

$$
2 g(H)-2=H^{2}+H \cdot K_{X} \geq H^{2}+H \cdot K_{i}=H^{2}+H \cdot K_{X}-2 H \cdot D,
$$

i.e, $D \cdot H \geq 0$. Thus by the duality of the closure of the effective cone $\overline{\mathrm{NE}}(X)$ and the nef cone, we conclude that $D \in \overline{\mathrm{NE}}(X)$ [15, Proposition 2.3]. By property (iii), we also have $C:=\left(K_{X}+K_{i}\right) / 2 \in \overline{\mathrm{NE}}$. Rewriting we get $K_{X}=C+D$ and $K_{i}=C-D$ as claimed. Note that nothing is gained by applying the inequality to other smooth connected divisors $C$ with $C^{2} \geq 0$, since such divisors are nef. Finally note that $H \cdot K_{i}=2 g(H)-2-H^{2}=H \cdot K_{X}$ if and only if $D=0$, since $\overline{\mathrm{NE}} \cap H^{\perp}=(0)$. This proves Theorem 2 up to algebraicity.

To prove that the KM classes are of type $(1,1)$, we need a slight generalization of [2, Proposition 3.1]. For simplicity we restrict ourselves to the $\mathrm{SU}(2)$ case and a statement about Hodge types, but the proof can easily be modified to show that all $\mathrm{SO}(3)$ polynomials $q_{L, k}(X)$ with $L \in \mathrm{NS}(X)$ 
come from an algebraic cycle (cf. [loc. cit.]). Consider the Donaldson polynomial $q_{d}$ as an element of $S^{d} H^{2}(X)$ endowed with its natural Hodge structure.

Lemma 6. For every $d \geq 0$, the Donaldson polynomials $q_{d}(X)$ are pure of type $(d, d)$.

Proof. We temporarily index the polynomials on $H_{2}(X)$ by $k=c_{2}$. By [2, Proposition 3.1] the lemma is true for $q_{k}$ with odd $k \gg 0$ and evaluated on two-dimensional classes. Here sufficiently large means that the moduli space $\mathscr{M}_{k}(X)$ of stable bundles on $X$ for generic polarization $H$ is generically smooth and reduced of the proper dimension, and that the lower moduli spaces $\mathscr{M}_{k^{\prime}}(X)$ for $k^{\prime}<k$ have sufficiently low dimension, so that we can apply Morgan's comparison result [11]. For the general case we use stabilization.

By [12, Theorem 2.1.1], for every $k$ we can find an $l_{0}$, and $\epsilon_{1}, \ldots, \epsilon_{l}$ sufficiently small such that for a generic polarization on the $l \geq l_{0}$-fold blowup of type $H-\sum \epsilon_{i} E_{i}$, the moduli space $\mathscr{M}_{k+l}\left(\hat{X}\left(x_{1}, \ldots, x_{l}\right)\right)$ is generically smooth of the proper dimension, and the lower moduli spaces $\mathscr{M}_{k^{\prime}}(\hat{X})$ with $k^{\prime}<k$ have sufficiently low dimension. Thus $q_{k+l}(\hat{X})$ is pure of type $(d+4 l, d+4 l)$.

Now by the blowup formula [5, Theorem 4.3.1], we have

$$
q_{k}(X)=\left(-\frac{1}{2}\right)^{l} q_{k+l}\left(\hat{X}\left(x_{1}, \ldots x_{l}\right)\right)\left(E_{1}^{4}, \ldots, E_{l}^{4},-\right) .
$$

Clearly the exceptional divisors $E_{i}$ are pure of type $(1,1)$, hence $q_{k}(X)$ is pure of type $(d, d)$. Finally

$$
q_{k}(X)(p t,-)=-\frac{1}{2} q_{k+1}(\hat{X}(x))\left(E^{6},-\right),
$$

by [5, below Corollary 4.3.2] (without proof) or [14], and so we can use the same argument as above. q.e.d.

The rest of the proof is now straightforward. Let

$$
C(X)=e^{-Q / 2} q(X)=\sum a_{i} e^{K_{i}} .
$$

Then $C(X)=\sum C_{d}(X)$, where $C_{d}(X)$ is a homogeneous polynomial of pure type $(d, d)$, since $\exp (-Q / 2)$ as well as $q(X)$ is a sum of homogeneous polynomials of pure type. Write $K_{i}=\alpha_{i}+\beta_{i}+\bar{\alpha}_{i}$, with $\alpha_{i} \in H^{0,2}$, $\beta_{i} \in H^{1,1}$. Let $z \in H^{1,1}$ be variable and denote by $\frac{\partial}{\partial z}$ the directional derivative. Then

$$
\frac{\partial^{n} C(X)}{\partial z^{n}}=\sum_{d=0}^{\infty} \frac{\partial^{n} C_{d}}{\partial z^{n}}=\sum a_{i}\left\langle\beta_{i}, z\right\rangle^{n} e^{K_{i}}
$$


Clearly $\frac{\partial^{n} C_{d}}{\partial z^{n}}$ has pure type $(d-n, d-n)$. Thus, upon restriction to $H^{0,2}(X)$ only the constant term contributes, and we get

$$
\left.\frac{\partial^{n} C(X)}{\partial z^{n}}\right|_{H^{0,2}}=\text { constant }=\sum a_{i}\left\langle\beta_{i}, z\right\rangle^{n} e^{\bar{\alpha}_{i}}
$$

We conclude that

$$
\sum_{\alpha_{i} \neq 0} a_{i}\left\langle\beta_{i}, z\right\rangle^{n} e^{\bar{\alpha}_{i}}=0 .
$$

Since $z$ and $n$ are arbitrary, we find that the sum must be empty, i.e., all KM classes $K_{i}$ are of type $(1,1)$, and we are done.

\section{Acknowledgements}

I would like to thank Professor Schneider for inviting me to the Graduirtenkolleg "Komplexe Mannigfaltigkeiten" Bayreuth, which supported me while working on this article. Stefan Bauer and Andreas Steffens are thanked for some useful remarks. Finally, thanks to the members of the Mathematisches Institut Bayreuth for providing a friendly and stimulating atmosphere.

\section{Bibliography}

[1] W. Barth, C. Peters \& A. Van de Ven, Compact complex surfaces, Springer, Berlin, 1984.

[2] R. Brussee, On the (-1) curve conjecture of Friedman and Morgan, Invent. Math. 114 (1993) p. 219-229.

[3] R. Fintushel \& R. Stern, Immersed 2-spheres in 4-manifolds and the structure of the Donaldson invariant for 4-manifolds of simple type, in preparation.

[4] R. Friedman \& J. W. Morgan, Algebraic surfaces and 4-manifolds, Bull. Amer. Math. Soc. 18 (1988) 1-19.

[5] R. Friedman \& J. W. Morgan, Smooth four-manifolds and complex surfaces, Ergebnisse Math. Grenzgeb. 3, Folge, Springer, Berlin, 27 (1994).

[6] P. Griffiths \& J. Harris, Principles of algebraic geometry, Wiley, New York, 1978.

[7] P. B. Kronheimer, The genus minimizing property of algebraic curves, Bull. Amer. Math. Soc. 29 (1993) 63-69.

[8] P. B. Kronheimer \& T. S. Mrowka, Recurrence relations and asymptotics for fourmanifold invariants, Bull. Amer. Math. Soc. 30 (1994) 215-221.

[9] J. Li, Algebraic geometric interpretation of Donaldson's polynomial invariants, J. Differential Geometry 37 (1993) 417-466.

[10] __ Kodaira dimension of moduli spaces of vector bundles of surfaces, Invent. Math. 115 (1994) $1-40$. 
[11] J. W. Morgan, Comparison of the Donaldson polynomial invariants with their algebrogeometric analogues, Topology 32 (1993) 449-488.

[12] J. W. Morgan \& K. G. O'Grady, Differential topology of complex surfaces, Lecture Notes in Math., Vol. 1545, Springer, Berlin, 1993.

[13] K. O'Grady, Algebro-geometric analogues of Donaldson's polynomials, Invent. Math. 107 (1992) 351-359.

[14] P. S. Oszvath, Some blowup formulas for SU(2) Donaldson polynomials, preprint, Princeton, October 1993.

[15] P. Wilson, Towards birational classification of algebraic varieties, Bull. London Math. Soc. 19 (1987) 1-48.

FakUltät Für Mathematik Universität Bielefeld, Postfach 
